Pacific Journal of Mathematics

CONGRUENCE CONDITIONS ON INTEGERS REPRESENTED 


\title{
CONGRUENCE CONDITIONS ON INTEGERS REPRESENTED BY TERNARY QUADRATIC FORMS
}

\author{
A. G. EARNEST
}

\begin{abstract}
A relationship is proved between certain integers $c$ primitively represented by a spinor genus of integral ternary quadratic forms and integers of the type $c t^{2}$ primitively represented by individual forms in the spinor genus. This relationship is shown to explain various representation properties observed in the literature.
\end{abstract}

There are numerous examples in the literature of pairs of integral ternary quadratic forms which lie in the same genus but which primitively represent different sets of integers according to certain congruence properties. For instance, consider the forms $f=x^{2}+x y+y^{2}+9 z^{2}$ and $g=x^{2}+3\left(y^{2}+y z+z^{2}\right)$, which are representatives of the two equivalence classes of a genus. $f$ primitively represents an integer of the form $4 t^{2}, t>0$, if and only if $t \equiv-1(\bmod 3)$, while $g$ primitively represents such an integer if and only if $t \equiv 1(\bmod 3)$ (see [12]).

Recent work of Peters [10] indicates that the above-illustrated phenomenon is a consequence of the splitting of the genus into spinor genera. Much progress has been made in the study of spinor genus representations in the work of several authors which will be cited in detail below. However, for definite forms, for which there may be many equivalence classes within a single spinor genus, there remains the problem of relating the representations by the individual forms in the spinor genus to the representations by the spinor genus as a whole. It is this problem that we address in the present paper. The main result, appearing as Theorem 2.3, shows that under certain conditions a relationship holds between integers $c$ primitively represented by a spinor genus and integers of the type $c t^{2}$ primitively represented by the forms in the spinor genus.

The behavior discussed here is unique to ternary forms in light of two major results. First, definite forms in 4 or more variables (primitively) represent all sufficiently large integers which are (primitively) represented by their genus. For a proof of this theorem, and its history, see [1]. Extensions of this result to representations of forms by forms, and to representations by quadratic lattices over number fields, are found in [3]. Secondly, indefinite forms in 4 or more variables (primitively) represent all integers (primitively) represented by their genus (see e.g., [12], Theorem 53). As the equivalence class and spinor genus of such indefinite forms 
coincide, this latter result is a special case of general theorems on spinor genus representations.

1. Preliminaries. Throughout the present paper we will adopt the geometric language of quadratic spaces and lattices. Unexplained notations and terminology will follow that of [9]. Let $(V, Q)$ be a 3 -dimensional quadratic space over the field $\boldsymbol{Q}$ of rational numbers. All lattices considered will be $Z$-lattices on $V$ for which $Q(v) \in Z$ for vectors $v$ in the lattice.

The objects of study here will be the sets of integers $Q(L)$, $Q(\operatorname{gen}(L))$, and $Q(\operatorname{spn}(L))$ which are represented by a lattice $L$, the genus gen $(L)$ of $L$, and the spinor genus $\operatorname{spn}(L)$ of $L$, respectively. The corresponding sets for integers primitively represented will be denoted by $Q^{*}(L), Q^{*}(\operatorname{gen}(L))$, and $Q^{*}(\operatorname{spn}(L))$, respectively. The following result relating $Q(L)$ and $Q(\operatorname{gen}(L))$ is well-known and is independent of the rank of $L$ except to assume that the rank is at least 3.

Proposition 1.1. (i ) $c \in Q($ gen $(L))$ if and only if $c t^{2} \in Q(L)$ for some integer $t$ which is prime to $2 d$ (d denotes the discriminant of $L)$.

(ii) $c \in Q^{*}(\operatorname{gen}(L))$ if and only if $c t^{2} \in Q^{*}(L)$ for some integer $t$ which is prime to $2 d$.

The remainder of this paper is largely an attempt to obtain results of a similar nature relating those integers $c$ (primitively) represented by $\operatorname{spn}(L)$ and those integers of the form $c t^{2}$ (primitively) represented by $L$ itself.

Let $J_{\boldsymbol{Q}}$ denote the full idèle group over $\boldsymbol{Q}, P_{D}$ the subgroup of principal idèles generated by elements of $D=\theta\left(O^{+}(V)\right.$ ) (where $\theta$ denotes the spinor norm mapping), and $J_{Q}^{L}$ the subgroup of all $j$ for which $j_{p} \in \theta\left(O^{+}\left(L_{p}\right)\right)$ for all finite primes $p$. Let $\Sigma$ be an element of $J_{V}$, the group of split rotations on $V$. Take $i$ to be an idèle satisfying $i_{p} \in O(\Sigma)$ for all $p$. If $j$ is another such idèle, then $j \in i J_{Q}^{2}$. So the map given by sending $\Sigma$ to the canonical image of $i$ in $J_{Q} / J_{Q}^{2}$ is well-defined; we denote it also by $\theta$.

For an integer $t$ define an idèle $j(t)$ componentwise by

$$
j(t)_{p}= \begin{cases}1 & \text { if } \operatorname{ord}_{p} t \text { is even, or } p=\infty \\ p & \text { if } \operatorname{ord}_{p} t \text { is odd. }\end{cases}
$$

Proposition 1.2. If $c t^{2} \in Q^{*}(L)$ for some $t$ prime to $2 d$, then $c \in Q^{*}(\Sigma L)$ for some $\Sigma \in J_{V}$ with $j(t) \in \theta(\Sigma)$. 
Proof. Let $v$ be a primitive vector of $L$ so that $Q(v)=c t^{2}$. For $p \mid t$ there exist bases $x_{1}^{p}, x_{2}^{p}, x_{3}^{p}$ for $L_{p}$ such that the matrix of $Q$ is $\left(\begin{array}{lr}0 & 1 / 2 \\ 1 / 2 & 0\end{array}\right) \perp\langle-4 d\rangle$, and for which $v=\sum a_{i}^{p} x_{i}^{p}$ with $p^{2 s} \| a_{1}^{p}$ where $s$ is the exact power of $p$ dividing $t$. Modifying the last basis vector if necessary by a local unit, we can approximate the local bases over all prime divisors of $t$ by a global basis $x_{1}, x_{2}, x_{3}$ for $L$ see [1], p. 33 and [3], Lemma 1.6). Writing $v=\sum a_{i} x_{i}$ we have $p^{2 s} \| a_{1}$ if the approximation is sufficiently good locally. Consider the lattice

$$
K=t \boldsymbol{Z} x_{1}+t^{-1} \boldsymbol{Z} x_{2}+\boldsymbol{Z} x_{3} .
$$

The vector $t^{-1} v$ is a primitive vector of $K$ for which $Q\left(t^{-1} v\right)=c$. Now locally $K_{p}$ is given by

$$
K_{p}= \begin{cases}L_{p} & \text { for } p \nmid t \\ \sigma_{p} L_{p} & \text { for } p \mid t,\end{cases}
$$

where $\sigma_{p}$ is the local isometry defined by $x_{1}^{p} \rightarrow p^{s} x_{1}^{p}, x_{2}^{p} \rightarrow p^{-s} x_{2}^{p}$ and $x_{3}^{p} \rightarrow x_{3}^{p}$. The spinor norm of $\sigma_{p}$ is equal to $p$. We obtain $K=\Sigma L$ and $j(t) \in \theta(\Sigma)$.

CoROllaRy 1.3. If $c t^{2} \in Q^{*}(L)$ for some $t$ prime to $2 d$ for which $j(t) \in P_{D} J_{Q}^{L}$, then $c \in Q^{*}(\operatorname{spn}(L))$.

Proof. $\Sigma L$ lies in $\operatorname{spn}(L)$ if and only if $\theta(\Sigma) \in P_{D} J_{Q}^{L}$.

The above corollary is not true if $Q^{*}$ is replaced by $Q$ throughout, as is shown by the following example, which was pointed out to the author by Professor Jones, who attributes it to G. Pall.

EXAMPLE 1.4. Consider the forms $f=x^{2}+y^{2}+16 z^{2}$ and $g=$ $2 x^{2}+2 y^{2}+5 z^{2}+2 x z+2 y z$. Each form lies in a one-class spinor genus and their genus consists precisely of these two classes. $f$ primitively represents $m^{2}, m$ odd, $m>0$, if and only if $m \equiv 1(\bmod 4)$; $g$ primitively represents such $m^{2}$ if and only if $m \equiv 3(\bmod 4)$. For example, $g(1,0,1)=3^{2}$ and so $g(3,0,3)=9^{2}$. Certainly $j(9) \in P_{D} J_{Q}^{L_{\text {. }}}$ but $g$ does not represent 1 .

However, the converse of Corollary 1.3 is true with $Q^{*}$ replaced by $Q$. This is easily seen by examining the corresponding matrices and we state it here without proof.

Proposition 1.5. If $c \in Q(\operatorname{spn}(L))$, then $c t^{2} \in Q(L)$ for some $t$ prime to $2 d$ for which $j(t) \in P_{D} J_{Q}^{L}$.

2. Main results. If $\mathscr{G}$ is a genus of forms of rank 4 or more 
and $c \in Q(\mathscr{G})$, then it follows that $c \in Q(\mathscr{S})$ for every spinor genus $\mathscr{S}$ contained in $\mathscr{G}$. However, if the rank is 3 one can conclude only that $c \in Q(\mathscr{S})$ either for all $\mathscr{S} \subseteq \mathscr{G}$ or for exactly half. This was shown for indefinite forms over the rationals by Jones and Watson [6], for lattices over an algebraic number field it is a consequence of work of Kneser [7], and a corresponding result for higher dimensional representations was obtained by Hsia [2]. The situation for primitive representations is analogous.

In this section $\mathscr{G}$ will denote a genus of ternary lattices. We will call an integer $c$ a (primitive) spinor-exception for $\mathscr{G}$ if $c$ is (primitively) represented by $\mathscr{G}$ but not by some spinor genus in $\mathscr{G}$. Note that the "exceptional integers" of Watson [12], Ch. 7, are the primitive spinor-exceptional integers in our terminology. Necessary and sufficient conditions for (primitive) spinor-exceptionality have been determined by Schulze-Pillot [11], extending the techniques of [2]. For later reference, we record these conditions here.

Let $c \in Q(\mathscr{G})$ where $\mathscr{G}$ is a genus of ternary lattices of discriminant $d$. Let $E=\boldsymbol{Q}(\sqrt{m})$ where $m$ is that squarefree integer in the rational square class of $-c d$. For any prime $p$, let $N_{p}$ denote the group of local norms $N_{E_{P} / Q_{p}}\left(\dot{E}_{P}\right)$, where $P$ is a prime ideal of $E$ lying over $p$. If $(,)_{p}$ denotes the Hilbert symbol, an alternative characterization of $N_{p}$ is $N_{p}=\left\{x \in \dot{\boldsymbol{Q}}_{p}:(x, m)_{p}=1\right\}$.

Let $L$ be a lattice in $\mathscr{G}$. The integer $c$ is a spinor-exception for $\mathscr{G}$ if and only if the following conditions hold:
$(*)$
(i) $-c d \notin \grave{\mathbf{Q}}^{2}$
(ii) $\theta\left(0^{+}\left(L_{p}\right)\right) \subseteq N_{p}$
(iii) $\theta\left(L_{p}, c\right)=N_{p}$,

the second condition holding for all $p$ (with the convention $L_{\infty}=V_{\infty}$ ) and the third for finite $p$. We will not need to compute the groups $\theta\left(L_{p}, c\right)$ explicitly, so we do not give their definition here (see [11]). The necessity of conditions (i) and (ii) was observed already in [6], [7], and [2]. For primitive spinor-exceptionality, one needs only to replace $\theta\left(L_{p}, c\right)$ by a slightly different group $\theta^{*}\left(L_{p}, c\right)$. It is useful to note here that the only prime divisors of $m$ must be divisors of $2 d$ since $\theta\left(0^{+}\left(L_{p}\right)\right)$ consists precisely of the unit square classes for all other primes $p$, and so condition (ii) forces those primes to be unramified in $E / Q$.

The conditions given above guarantee that the group index $\left(J_{Q}: P_{D} N J_{Q}^{L}\right)$ equals 2 , where $N=\left\{j \in J_{Q}: j_{p} \in N_{p}\right.$ for all $\left.p\right\}$. We say that a primitive spinor-exceptional $c$ divides the spinor genera in $\mathscr{G}$ into two $c$-half-genera. If $c \in Q^{*}(\operatorname{spn}(L))$ for such an integer $c$, then $c \in Q^{*}(\operatorname{spn}(\Sigma L))$ if and only if $\theta(\Sigma)=j J_{Q}^{2}$ with $j \in P_{D} N J_{Q}^{L}$ (i.e., under these conditions $L$ and $\Sigma L$ lie in the same $c$-half-genus).

For the idèles $j(t)$ introduced in $\S 1$, this last condition can be expressed concretely in terms of the integer $t$. We denote by (-) 
the usual Jacobi symbol.

Lemma 2.1. Suppose $c$ is a primitive spinor-exception for gen $(L)$ and let $E=\boldsymbol{Q}(\sqrt{m})$ and $N$ be as above. Let $t$ be a positive integer prime to $2 d$. Then $j(t) \in P_{D} N J_{Q}^{L}$ if and only if $(m / t)=1$.

Proof. For any $j \in J_{Q}$, it is a consequence of the Hilbert Reciprocity Law that $j \in P_{\mathbf{Q}} N$ if and only if $\Pi\left(j_{p}, m\right)_{p}=1$, the product taken over all primes $p$. By the definition of $j(t)$ and the condition $\operatorname{gcd}(t, 2 d)=1$ (hence also $\operatorname{gcd}(t, m)=1$ ), this product reduces to the product taken over all primes $p$ for which ord ${ }_{p} t$ is odd, and this latter product is equal to the Jacobi symbol $(m / t)$. So the proof will be complete if we show that $j(t) \in P_{D} N J_{Q}^{L}$ is equivalent to $j(t) \in P_{Q} N$. In fact, we will show this to be the case for any $j \in J_{Q}$ for which $j_{\infty}>0$.

If $D=Q$, then $P_{Q} N \leqq P_{D} N J_{Q}^{L}$ and equality of the subgroups follows since both have index 2 in $J_{\boldsymbol{Q}}$. Otherwise $D=\boldsymbol{Q}^{+}$. By condition (ii) of $\left(^{*}\right)$ we have $P_{D} N J_{Q}^{L}=P_{D} N I$, where $I=\left\{i \in J_{Q}: i_{p}=1\right.$ for all finite $p\}$. So $j \in P_{D} N I$ implies that $j \in P_{D} N \cong P_{Q} N$ when $j_{\infty}>0$. Conversely, suppose $j \in P_{Q} N$ and $j_{\infty}>0$. So there exists $b \in Q$ such that $\left(b j_{p}, m\right)_{p}=1$ for all $p$. If $b>0$ then $j \in P_{D} N$ and there is nothing to prove. So assume that $b<0$. Consequently, $m>0$ since $1=\left(b j_{\infty}, m\right)_{\infty}=(b, m)_{\infty}$. By the Chinese Remainder theorem and Dirichlet's theorem on primes in an arithmetic progression, there exists a prime $p_{0}$ such that $p_{0} \equiv-1(\bmod 8 m)$. This assures that $\left(-p_{0}, m\right)_{p}=1$ for all $p \neq p_{0}$. So by Hilbert Reciprocity it follows that also $\left(-p_{0}, m\right)_{p_{0}}=1$. This gives

$$
\left(-p_{0} b j_{p}, m\right)_{p}=\left(-p_{0}, m\right)_{p}\left(b j_{p}, m\right)_{p}=1
$$

for all $p$, and $-p_{0} b \in D$ as desired.

Lemma 2.2. Suppose that $c$ is a primitive spinor-exception for $\mathscr{G}$ and $L \in \mathscr{G}$. If $c \in Q^{*}(\operatorname{spn}(L))$ and $c t^{2} \in Q^{*}(L)$ with $\operatorname{gcd}(t, 2 d)=1$ and $t>0$, then $(m / t)=1$.

Proof. It follows from the assumptions and Proposition 1.2 that $c \in Q^{*}(\operatorname{spn}(\Sigma L))$ where $j(t) \in \theta(\Sigma)$. If $(m / t)$ were equal -1 then $j(t) \notin$ $P_{D} N J_{Q}^{L}$ and $\operatorname{spn}(\Sigma L)$ would lie in the $c$-half-genus of $\mathscr{G}$ opposite from $L$. Then $c \notin Q^{*}(\operatorname{spn}(L))$ contrary to assumption.

We now state and prove a partial spinor genus analogue to Proposition 1.1.

THEOREM 2.3. Suppose that $c$ is a primitive spinor-exception 
for $\mathscr{G}$ and $L \in \mathscr{G}$. Then $c \in Q^{*}(\operatorname{spn}(L))$ if and only if $c t^{2} \in Q^{*}(L)$ for some $t$ with $t>0, \operatorname{gcd}(t, 2 d)=1$, and $(m / t)=1$.

Proof. If $c \in Q^{*}(\operatorname{spn}(L))$ then $c t^{2} \in Q^{*}(L)$ for some $t$ with $t>0$ and $\operatorname{gcd}(t, 2 d)=1$ by Proposition 1.1. That $(m / t)=1$ then follows from Lemma 2.2. Conversely, if $c t^{2} \in Q^{*}(L)$ with $t>0, \operatorname{gcd}(t, 2 d)=1$ and $(m / t)=1$, then $c$ is primitively represented by the $c$-half-genus of $\operatorname{spn}(L)$, hence by $\operatorname{spn}(L)$ itself.

We will illustrate the results obtained above by using them to analyze three specific examples from the literature. In each case the first step in the procedure is the identification of a primitive spinor-exceptional integer $c$. As the verifications of the conditions of $\left(^{*}\right)$ are routine for these examples, we will omit them.

EXAMPle 2.4. Consider the forms $f=x^{2}+x y+y^{2}+9 z^{2}$ and $g=x^{2}+3\left(y^{2}+y z+z^{2}\right) . \quad f$ and $g$ are representatives of the two spinor genera of a genus, and 4 is a primitive spinor-exception for the genus. In this case, $c=4, d=27 / 4$ and $m=-3$. Since $4 \epsilon$ $Q^{*}(\operatorname{spn}(g))$ it follows from Lemma 2.2 that $4 t^{2} \in Q^{*}(g)$ with $t>0$ and $\operatorname{gcd}(t, 6)=1$ holds only for $t$ such that $((-3) / t)=1$ or, by quadratic reciprocity, $t \equiv 1(\bmod 3)$. Moreover, by Theorem 2.3 $4 t^{2} \in Q^{*}(f)$ for such integers $t$ only when $t \equiv-1(\bmod 3)$. In this specific example one can say more. Since all integers of the form $4 t^{2}, \operatorname{gcd}(t, 6)=1$, are represented primitively by the genus and $f$ and $g$ are representatives of the only two equivalence classes in the genus, each of $f$ and $g$ must primitively represent all such integers which are permitted by the congruence conditions.

EXAMPLE 2.5. (see [10], p. 77) $f=x^{2}+2 y^{2}+64.17 z^{2}$ and $g=$ $(2 x+z)^{2}+2 y^{2}+16.17 z^{2}$ are representatives of the two spinor genera of a genus, and 17 is a primitive spinor-exception. Now $c=17$, $d=2^{7} .17$ and $m=-2$. For $t>0, \operatorname{gcd}(t, 34)=1,17 t^{2}$ lies in $Q^{*}(f)$ only when $((-2) / t)=1$; that is, when $t \equiv 1$ or $3(\bmod 8)$ (note that $f(3,2,0)=17$, so that $\left.17 \in Q^{*}(\operatorname{spn}(f))\right)$. Similarly, such $17 t^{2}$ lie in $Q^{*}(g)$ only when $t \equiv-1$ or $-3(\bmod 8)$.

EXAMPle 2.6. (see [5], p. 191) $f=x^{2}+3 y^{2}+36 z^{2}$ and $g=3 x^{2}+$ $4 y^{2}+9 z^{2}$ are representatives of the two spinor genera of a genus with 1 as a primitive spinor-exceptional integer. Here $c=1, d=108$ and $m=-3$. Since $1 \in Q^{*}(f)$ we have $t^{2} \in Q^{*}(f)$ implies $t \equiv 1(\bmod 3)$ for $t>0, \operatorname{gcd}(t, 6)=1$, and $t^{2} \in Q^{*}(g)$ implies $t \equiv-1(\bmod 3)$. As in Example 2.4, we can again draw the stronger conclusion that all of these integers are indeed primitively represented by the appropriate 
form. We note also that Jones and Pall determined all integers represented (not necessarily primitively) by these forms. In fact, $f$ is a "regular" form, that is, $Q(f)=Q(\operatorname{gen}(f))$, and $Q(g)=$ $Q($ gen $(g))-\left\{t^{2}: t>0\right.$ and all prime factors of $t$ are $\left.\equiv 1(\bmod 3)\right\}$.

REMARK 2.7. As there are not effective spinor-generic invariants known at the present time, we note here that in special cases primitive representations can be used for the purpose of determining the spinor genus of a given form. Observe first that the identification of primitive spinor-exceptional integers, if any, for a genus is essentially a local problem due to the nature of the conditions $\left(^{*}\right)$. Assuming the existence of such an integer $c$, one can determine whether two given forms in the genus lie in the same $c$-half-genus by producing single integers of the type $c t^{2}$, with $\operatorname{gcd}(t, 2 d)=1$, primitively represented by the forms. In the special case when there are only two spinor genera in the genus, this gives a procedure for determining to which spinor genus a given form belongs.

3. The role of exceptional integers. It was mentioned in our introductory remarks that the work of Peters in [10] indicates that the representation properties studied in this paper are the results of the splitting of the genus into spinor genera. More specifically, it is shown there under the assumption of a suitable generalized Riemann hypothesis that a positive definite ternary form in a genus consisting of a single spinor genus (primitively) represents all sufficiently large integers (primitively) represented by its genus. The methods employed in that paper follow those used extensively by Linnik and Malyshev in work which is described in [8].

The arguments in fact show that a positive definite ternary form whose genus contains more than one spinor genus still represents all sufficiently large integers represented by its genus with the possible exception of those which satisfy the condition (ii) of $\left(^{*}\right.$ ) for all finite primes $p$. Note that if an integer $c$ violates such a condition, then every integer of the type $c t^{2}$ does so as well. In that case all sufficiently large integers of this type are represented by every form in the genus. There is thus a gap between this theory and the present work in that nothing is said by either concerning those integers $c t^{2}$ where $c$ is not a primitive spinor-exception but which still satisfies (ii) of $\left(^{*}\right)$ for all finite primes $p$. Peters comments ([10], p. 77) that he does not know whether the results of his paper remain true if only the primitive spinor-exceptional integers are excluded rather than the larger set.

From the approach taken in this paper it is natural to ask whether there are cases of ternary genera having more than one 
spinor genus but for which there are no primitive spinor-exceptional integers. We close this section with two examples showing that this can indeed be the case, both for definite and indefinite forms. Note that the second example shows a pair of inequivalent indefinite forms which lie in the same genus and primitively represent the same set of integers.

EXAMPLE 3.1. The genus of the form $f=x^{2}+17 y^{2}+17^{2} z^{2}$ contains two spinor genera. Consider the possible extensions $E$ that could arise from spinor-exceptional integers $c$. Since $f$ is positive definite the value of $m$ occurring must be negative. By the condition (ii) of $\left(^{*}\right)$ the only finite prime that can ramify in $E$ is 17 . But 2 ramifies in both $Q(\sqrt{-1})$ and $\boldsymbol{Q}(\sqrt{-17})$, thus eliminating all potential $E$ 's. Note that not only is no $c \in Q^{*}(\operatorname{gen}(f))$ spinor-exceptional, but none satisfies (ii) of (*). So by the above remarks $c \in$ $Q^{*}(f)$ for all sufficiently large $c$ from $Q^{*}($ gen $(f))$.

EXAMPLE 3.2. The genus of the indefinite form $f=-x^{2}+p_{0}^{2} y^{2}+$ $p_{\mathrm{c}}^{4} z^{2}$ with $p_{0} \equiv 1(\bmod 8)$ contains two spinor genera. If $c \in Q^{*}(\operatorname{gen}(f))$, the corresponding extension is $E=\boldsymbol{Q}(\sqrt{c})$ since $d=-p_{0}^{6}$. To satisfy (i) and (ii) of $\left(^{*}\right), c$ is not a square and no finite prime different from $p_{0}$ ramifies in $E$. In addition, the condition at $\infty$ requires that $E$ is a real quadratic field. So $c$ must be of the form $p_{0} k^{2}$ for some integer $k$. A computation of the group $\theta^{*}\left(L_{p_{0}}, p_{0} k^{2}\right)$ for the corresponding lattice $L$ shows this group to be equal to $\dot{\boldsymbol{Q}}_{p_{0}}$ which is not equal to the group of local norms at $p_{0}$ since $p_{0}$ ramifies in $E=$ $\boldsymbol{Q}\left(\sqrt{p_{0}}\right)$. So the integers of the form $p_{0} k^{2}$ also fail to be spinorexceptional, thus eliminating all possibilities.

4. Residues modulo a prime. The study of ternary representation properties appears to have been the primary motivation behind the introduction of the notion of the "quasi-genus" by Jones in [4]. The definition of the quasi-genus depends upon the idea of a prime "exceptional" for a form $f$. Here the word exceptional is used in a sense different from that of Watson, and refers to the property that all integers which are prime to $2 d$ and occur as denominators of automorphs of $f$ are quadratic residues modulo this prime. A prime $p$ is said to have "property $A$ " for the ternary form $f$ if $f$ primitively represents some integer $c$, and whenever $f$ primitively represents $c s^{2}$ and $c t^{2}$, with $s$ and $t$ positive and prime to $2 d$, there holds $(s / p)=(t / p)$. For instance, the prime 3 has property $A$ for both forms $f$ and $g$ of Example 2.4.

REMARK 4.1. Suppose that $c$ is a primitive spinor-exception for 
a given genus, and suppose that the condition $(m / t)=1$ reduces via quadratic reciprocity to a residue condition on $t$ modulo a single prime $p_{0}$. Given any $f$ in the genus, there exists some $t$ prime to $2 d$ so that $c t^{2} \in Q^{*}(f)$. The sign of $\left(t / p_{0}\right)$ depends upon whether or not $c \in Q^{*}(\operatorname{spn}(f))$. If also $a t^{2} k^{2} \in Q^{*}(f)$ with $\operatorname{gcd}\left(k, 2 p_{0} d\right)=1$, then $\left(t k / p_{0}\right)$ has the same sign as $\left(t / p_{0}\right)$; thus, $\left(k / p_{0}\right)=1$ for any such $k$. Hence, $p_{0}$ has property $A$ for every form in the genus.

REMARK 4.2. Jones proves ([4], Theorem 20) that if $p$ has property $A$ for a ternary form $f$, then $p$ is exceptional or semiexceptional (slightly different residue conditions are satisfied) for $f$. As anticipated by Jones ([4], p. 411), the converse of this theorem is false as shown by the examples of the preceding section. We need only note that 17 is an exceptional prime for each of the forms $f$ given in Examples 3.1 and 3.2, as can be seen by the conditions of [4], Theorem 5 .

\section{REFERENCES}

1. J.W.S. Cassels, Rational Quadratic Forms, Academic Press London, 1978.

2. J.S. Hsia, Representations by spinor genera, Pacific J. Math., 63 (1976), 147-152.

3. J.S. Hsia, Y. Kitaoka and M. Kneser, Representations of positive definite quadratıc forms, J. reine angew. Math., 301 (1978), 132-141.

4. B. W. Jones, Quasi-genera of quadratic forms, J. Number Theory, 9 (1977), 393-412.

5. B. W. Jones and G. Pall, Regular and semi-regular positive ternary quadratic forms, Acta Math., 70 (1939), 165-191.

6. B.W. Jones and G. L. Watson, On indefinite ternary quadratic forms, Canad. J. Math., 8 (1956), 592-608.

7. M. Kneser, Darstellungsmasse indefiniter quadratischer Formen, Math. Zeit., 77 (1961), 188-194.

8. A.V. Malyshev, O predstavlenii celyh cisel polozitel'nymi kvadraticnymi formami, Trudy Mat. Inst. Steklov, 65 (1962).

9. O. T. O'Meara, Introduction to Quadratic Forms, Springer Berlin, 1973.

10. M. Peters, Darstellungen durch definite ternäre quadratische Formen, Acta Arith., 34 (1977), 57-80.

11. R. Schulze-Pillot, Darstellung von Zahlen durch Spinorgeschlechter ternärer quadratischer Gitter, Diplomarbeit, Göttingen, 1977.

12. G. L. Watson, Integral Quadratic Forms, Cambridge University Press, Cambridge, 1960.

Received September 18, 1979.

University of Southern California

Los Angeles, CA 90007 



\section{PACIFIC JOURNAL OF MATHEMATICS}

\section{EDITORS}

DONALD BABBITT (Managing Editor)

University of California

Los Angeles, CA 90024

Hugo RossI

University of Utah

Salt Lake City, UT 84112

C. C. MOORE and ANDREW OGG

University of California

Berkeley, CA 94720

\section{J. DugunduI}

Department of Mathematics

University of Southern California

Los Angeles, CA 90007

R. Finn and J. Milgram

Stanford University

Stanford, CA 94305

\section{ASSOCIATE EDITORS}
E. F. BeCKenBACH
B. H. NeumanN
F. WOLF
K. YosHIDA

\section{SUPPORTING INSTITUTIONS}

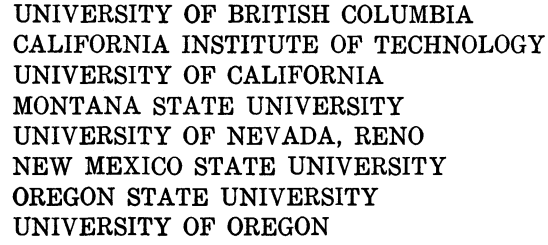

UNIVERSITY OF BRITISH COLUMBIA CALIFORNIA INSTITUTE OF TECHNOLOGY UNIVERSITY OF CALIFORNIA

MONTANA STATE UNIVERSITY

UNIVERSITY OF NEVADA, RENO

NEW MEXICO STATE UNIVERSITY

OREGON STATE UNIVERSITY UNIVERSITY OF OREGON

\author{
UNIVERSITY OF SOUTHERN CALIFORNIA \\ STANFORD UNIVERSITY \\ UNIVERSITY OF HAWAII \\ UNIVERSITY OF TOKYO \\ UNIVERSITY OF UTAH \\ WASHINGTON STATE UNIVERSITY \\ UNIVERSITY OF WASHINGTON
}

The Supporting Institutions listed above contribute to the cost of publication of this Journal, but they are not owners or publishers and have no responsibility for its content or policies.

Mathematical papers intended for publication in the Pacific Journal of Mathematics should be in typed form or offset-reproduced, (not dittoed), double spaced with large margins. Please do not use built up fractions in the text of the manuscript. However, you may use them in the displayed equations. Underline Greek letters in red, German in green, and script in blue. The first paragraph or two must be capable of being used separately as a synopsis of the entire paper. Please propose a heading for the odd numbered pages of less than 35 characters. Manuscripts, in triplicate, may be sent to any one of the editors. Please classify according to the scheme of Math. Reviews, Index to Vol. 39. Supply name and address of author to whom proofs should be sent. All other communications should be addressed to the managing editor, or Elaine Barth, University of California, Los Angeles, California, 90024.

50 reprints to each author are provided free for each article, only if page charges have been substantially paid. Additional copies may be obtained at cost in multiples of 50 .

The Pacific Journal of Mathematics is issued monthly as of January 1966. Regular subscription rate: $\$ 84.00$ a year (6 Vols., 12 issues). Special rato: $\$ 42.00$ a year to individual members of supporting institutions.

Subscriptions, orders for numbers issued in the last three calendar years, and changes of address shoud be sent to Pacific Journal of Mathematics, P.O. Box 969, Carmel Valley, CA 93924, U.S.A Old back numbers obtainable from Kraus Periodicals Co., Route 100, Millwood, NY 10546.

PUBLISHED BY PACIFIC JOURNAL OF MATHEMATICS, A NON-PROFIT CORPORATION

Printed at Kokusai Bunken Insatsusha (International Academic Printing Co., Ltd.). 8-8, 3-chome, Takadanobaba, Shinjuku-ku, Tokyo 160, Japan.

Copyright (C) 1980 by Pacific Jounal of Mathematics Manufactured and first issued in Japan 


\section{Pacific Journal of Mathematics \\ Vol. 90, No. $2 \quad$ October, 1980}

Frank Hayne Beatrous, Jr., Hölder estimates for the $\bar{\partial}$ equation with a support condition ..................................... 249

Charles L. Belna, Michael Jon Evans and Paul Humke, Planar continua

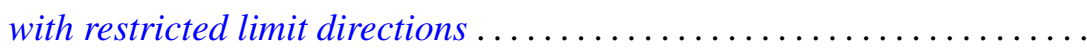

Leon Brown and Takashi Ito, Classes of Banach spaces with unique isometric preduals................................. 261

V. K. Deshpande, Completions of Noetherian hereditary prime rings ..... . 285

Deepak Dhar, Asymptotic enumeration of partially ordered sets . . . . . . . 299

Zeev Ditzian, On interpolation of $L_{p}[a, b]$ and weighted Sobolev

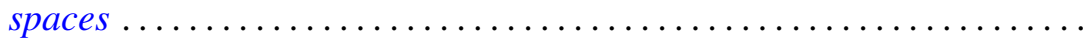

Andrew George Earnest, Congruence conditions on integers represented by

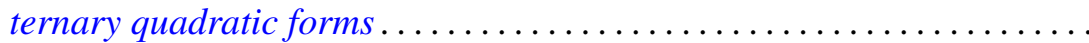

Melvin Faierman, Bounds for the eigenfunctions of a two-parameter system of ordinary differential equations of the second order ..............

Hector O. Fattorini, Vector-valued distributions having a smooth

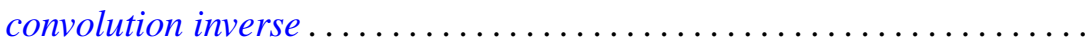

Howard D. Fegan, The spectrum of the Laplacian on forms over a Lie

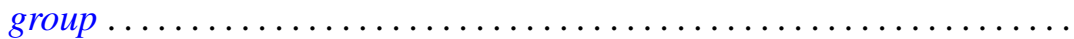

Gerald Leonard Gordon, On the degeneracy of a spectral sequence associated to normal crossings..

S. Madhavan, On bisimple weakly inverse semigroups ... 397

Françoise Mathot, On the decomposition of states of some

Roger McCann, Embedding asymptotically stable dynamical systems into radial flows in $l_{2}$

Michael L. Mihalik, Ends of fundamental groups in shape and proper homotopy...............................

Samuel Murray Rankin, III, Boundary value problems for partial functional differential equations .

Randy Tuler, Arithmetic sums that determine linear characters on $\Gamma(N)$

Jeffrey D. Vaaler, On linear forms and Diophantine approximation ..

G. P. Wene, Alternative rings whose symmetric elements are nilpotent or a right multiple is a symmetric idempotent. . 